\title{
Seminare / Séminaires / Seminari 2011
}

\section{Praxiseröffnung/-übernahme}

\section{Themen}

Juristische Aspekte (Praxisbewilligung, Zulassung zur Sozialversicherung, Vertragswesen), Gesellschaftsformen / Ehe- und Erbrecht (Trennung Privat- vom Geschäftsvermögen, Ehegüterstand, Erbschaftsplanung), Praxiseinrichtung (Inneneinrichtung, Kostenberechnung), Praxisadministration (Leistungserfassungsund Abrechnungssysteme), Unternehmensbewertung einer Arztpraxis (Berechnung und Beurteilung des Unternehmenswertes), Finanzierung der Arztpraxis (Businessplan, Kredite, Absicherungsmöglichkeiten), Versicherungen/Vorsorge/Vermögen (Personen- und Sachversicherungen, Vorsorgeplanung).

\section{Sponsoren}

Die Kosten werden durch diverse Sponsoren (siehe www.fmhservices.ch) gedeckt.

\section{Daten}

$\begin{array}{lll}\text { K04 } & \begin{array}{l}\text { Donnerstag, 1. September 2011 } \\ \text { 9.00-16.30 Uhr }\end{array} & \begin{array}{l}\text { Zürich } \\ \text { FMT }\end{array} \\ \text { K05 } & \begin{array}{l}\text { Donnerstag, 3. November 2011 } \\ \text { 9.00-16.30 Uhr }\end{array} & \begin{array}{l}\text { Basel } \\ \text { Hotel Victoria }\end{array}\end{array}$

\section{Praxisübergabe}

Das Seminar richtet sich an zukünftige Praxisübergeber/innen. Hinweis: Vor allem aus steuer- und vorsorgeplanerischer Sicht lohnt es sich, sich bereits frühzeitig (5-10 Jahre) mit diesem Thema auseinanderzusetzen.

\section{Themen}

Juristische Aspekte (Praxisübergabevertrag, allg. Vertragswesen, Übergabe der Krankengeschichten), Unternehmensbewertung einer Arztpraxis (Berechnung Inventarwert und Goodwill als Verhandlungsbasis), Versicherungen/Vorsorge/Vermögen (Übergabe/Auflösung von Versicherungsverträgen, Pensions- und Finanzplanung), Steuern (Steueraspekte bei der Praxisübergabe, Optimierung der steuerlichen Auswirkungen, Liquidations- und Grundstückgewinnsteuer, Bestimmung des optimalen Übergabezeitpunktes).

\section{Sponsoren}

Die Kosten werden durch diverse Sponsoren (siehe www.fmhservices.ch) gedeckt.

$\begin{array}{lll}\text { Daten } & & \\ \text { K09 } & \begin{array}{l}\text { Donnerstag, 8. September 2011 } \\ \text { 13.30-18.00 Uhr }\end{array} & \begin{array}{l}\text { Zürich } \\ \text { FMT }\end{array} \\ \text { K10 } & \begin{array}{l}\text { Donnerstag, 10. November 2011 } \\ \text { 13.30-18.00 Uhr }\end{array} & \begin{array}{l}\text { Basel } \\ \text { Hotel Victoria }\end{array}\end{array}$

\section{Finanz- und Steuerplanung}

\section{Themen}

Finanzplanung (Businessplan, Buchhalterische Massnahmen vor Praxiseröffnung/-übernahme, Standardkontenplan für Ärzte, System der doppelten Buchhaltung, EDV-unterstützte Buchführungslösung), Steuern (Steueraspekte bei Eintritt in die Selbständigkeit, Steuerfallen und Steuerrisiken, optimierte Steuerplanung).

\section{Sponsoren}

Die Kosten werden durch diverse Sponsoren (siehe www.fmhservices.ch) gedeckt.

\section{Datum}

K12 Donnerstag, 15. September 2011 Bern 13.30-18.00 Uhr Schmiedstube

\section{Röntgen in der Arztpraxis}

Das Seminar richtet sich an Ärztinnen und Ärzte mit bestehender Praxis und an solche, die vor einer Praxiseröffnung oder Praxisübernahme stehen. Das Seminar ist auf maximal 15 Teilnehmer/innen beschränkt.

\section{Themen}

Vom konventionellen zum digitalen Röntgen, Rentabilität Röntgen in der Arztpraxis, Neue Vorschriften, Evaluation und Beschaffung neuer oder gebrauchter Anlagen, Komplette Marktübersicht mit Preisen und Leistungskomponenten. Die Seminarteilnehmer erstellen und bearbeiten innerhalb des Seminars digitale Röntgenbilder und erhalten eine komplexe Dokumentation über alle Themen - ein Vademekum rund ums Röntgen.

\section{Kosten}

300 CHF (inkl. sämtlicher Kursunterlagen und Verpflegung).

\section{Datum}




\section{Tarifwerk TARMED - Einführungskurs}

Das Seminar richtet sich an Ärztinnen und Ärzte mit bestehender Praxis und an solche, die kurz vor einer Praxiseröffnung oder Praxisübernahme stehen. Der Einführungskurs vermittelt den Kursteilnehmern die Grundlagen des Tarifwerkes TARMED. Die Seminarteilnehmerzahl ist begrenzt.

\section{Themen}

Fakten (Gesetzliche und vertragliche Grundlagen), Struktur (Tarifbrowser, Grundstruktur des Tarifwerkes, Regelhierarchie, Leistungsblöcke, Leistungsgruppen), Generelle Interpretationen (Wichtigste generelle Interpretationen), Parameter einer Tarifposition (Alle Parameter einer Tarifposition), Tarifpositionen aus dem Kapitel 00 Grundleistungen (Diverse Tarifpositionen aus dem Kapitel 00 Grundleistungen), Praxislabor und Präsenzdiagnostik (Neue Analyseliste), Organisationen und Informationsquellen (www.tarmedsuisse.ch usw.).

Kosten

200 CHF (inkl. Kursunterlagen).

Datum

$\begin{array}{lll}\text { K61 Dienstag, 20. September } 2011 & \text { Olten } \\ & \text { 14.00-17.15 Uhr } & \text { Hotel Arte }\end{array}$

\section{Praxismarketing für Ärzte}

Das Seminar richtet sich an Ärztinnen und Ärzte, welche vor einer Praxiseröffnung stehen oder bereits eine Praxis führen.

\section{Themen}

Der Erfolg einer Arztpraxis ist unlösbar mit gutem Service und Kundenorientierung verbunden. Damit sind mehr als gute Umgangsformen gemeint. Echter Service geht tiefer und bietet den Patientinnen und Patienten - oder Kunden - einen direkten Nutzen. Aus einer Arztpraxis wird DIE Praxis.

Die Herausforderung ist nicht, Service für Patienten zu leisten, sondern Service für Patienten aus der Sicht des Patienten erlebbar zu machen. Dazu gehört, dass man seine Kunden versteht, auf ihre Bedürfnisse vorbereitet ist, sie um Informationen bittet, ihnen genau zuhört, Verantwortung für das eigene Tun übernimmt, über das normale Mass hinaus engagiert zu sein, Menschen zu überraschen, und das immer regelmässig und auf gleichbleibend hohem Niveau!

Das Wichtigste zu den Themen Telefon, Empfang, Teamentwicklung wird diskutiert. Die wichtigsten Werbemöglichkeiten besprochen. Nicht das Aussergewöhnliche wollen wir tun, sondern das Gewöhnliche aussergewöhnlich gut!

\section{Kosten}

300 CHF (inkl. sämtlicher Kursunterlagen und Verpflegungen)

\section{Datum}

K64

Mittwoch, 7. September 201 09.00-16.30 Uhr

Bern Schmiedstube

\section{Telefonseminar für MPA (bzw. Praxisteam)}

Das Seminar richtet sich an MPAs, welche im telefonischen Kundenkontakt stehen bzw. Auszubildende, welche im professionellen Telefonieren angeleitet werden sollen. Die «Visitenkarte» der Arztpraxis soll den modernen Anforderungen gerecht werden.

\section{Themen}

Die Medizinische Praxisassistentin ist die Visitenkarte der Praxis. Sie hat den ersten und den letzen Kontakt mit den Patienten und beeinflusst wesentlich das Image der Arztpraxis. MPAs repräsentieren die Unternehmenskultur, organisieren die Praxis und sind somit ein wesentlicher Bestandteil für den Unternehmenserfolg. "You never get a second chance to make a first impression."

Der erste Eindruck wird durch den ersten Kontakt geprägt! Und fast immer findet der erste Kontakt, den ein Patient zu seiner Arztpraxis aufnimmt, über das Telefon statt.

Das richtige Telefonieren, das gerade in der Arztpraxis von grosser Bedeutung ist, wird einem nicht in die Wiege gelegt, sondern muss erlernt werden.

\section{Kosten}

300 CHF (inkl. sämtlicher Kursunterlagen und Verpflegungen).

Praxisteams erhalten einen Rabatt von $20 \%$ pro Teilnehmer/in.

\section{Datum}

K6 Mittwoch, 2. November 2011 Bern 09.00-16.30 Uhr Schmiedstube

\section{Ouverture et reprise d'un cabinet médical}

Le séminaire est destiné aux médecins sur le point d'ouvrir ou de reprendre un cabinet médical.

\section{Contenu}

Business plan (préparation du plan de financement et crédit d'exploitation, financement par la banque), Aménagement (implantation, projet et concept d'aménagement, choix du mobilier, budget), Estimation d'un cabinet (inventaire et goodwill), Administration d'un cabinet médical (dans le cabinet, par la banque), Assurances (toutes les assurances à l'intérieur et autour du cabinet), Passage du statut de salarié à celui d'indépendant et fiscalité.

\section{Sponsors}

Les coûts sont pris en charge par divers sponsors (voir www.fmhservices.ch). 


$\begin{array}{lll}\text { Dates } & & \\ \text { K22 } & \begin{array}{l}\text { Jeudi } 1 \text { septembre } 2011 \\ 17.00-21.30 \mathrm{~h}\end{array} & \begin{array}{l}\text { Lausanne } \\ \text { World Trade Center }\end{array} \\ \text { K23 } & \begin{array}{l}\text { Jeudi } 10 \text { novembre } 2011 \\ 17.00-21.30 \mathrm{~h}\end{array} & \begin{array}{l}\text { Genève } \\ \text { Ramada Park Hôtel }\end{array}\end{array}$

\section{Remise d'un cabinet médical}

Le séminaire s'adresse aux médecins désirant remettre un cabinet médical.

\section{Contenu}

Aspects juridiques (autour du contrat de remise/ reprise), Estimation d'inventaire et goodwill d'un cabinet, Assurances (prévoyance, assurances à l'intérieur et autour du cabinet), Conséquences fiscales d'une remise.

\section{Sponsors}

Les coûts sont pris en charge par divers sponsors (voir www.fmhservices.ch).

$\begin{array}{lll}\text { Date } & \\ \text { K25 } & \text { Jeudi } 17 \text { novembre } 2011 & \begin{array}{l}\text { Genève } \\ \text { Ramada Park Hôtel }\end{array}\end{array}$

Apertura e rilevamento di uno studio medico Il seminario è destinato ai Sigg. medici in procinto di aprire o di rilevare uno studio medico.

\section{Contenuto}

Business plan (preparazione del piano di finanziamento e del credito d'esercizio, prestito bancario), Pianificazione (insediamento, progetto e pianificazione, scelta del mobilio, budget), Valutazione di uno studio medico (inventario e goodwill), Amministrazione di uno studio medico (interna allo studio, rapporti con la banca), Assicurazioni (tutte le assicurazioni necessarie interne ed esterne allo studio), Passaggio dallo stato di dipendente a quello di indipendente, fiscalità.

\section{Documentazione}

Basato su una check-list per l'apertura di uno studio medico, il raccoglitore, consegnato ad ogni partecipante, contiene tutte le informazioni utili durante e dopo il seminario.

\section{Sponsor}

Diversi sponsor si fanno carico delle spese (si rimanda al sito www.fmhservices.ch).

Date

\begin{tabular}{|lll} 
K52 & $\begin{array}{l}\text { Mercoledì } 28 \text { settembre } 2011 \\
\text { dalle } 14.00 \text { alle } 18.00\end{array}$ & $\begin{array}{l}\text { Chiasso } \\
\text { FMH Fiduciaria } \\
\text { Services }\end{array}$ \\
K53 & $\begin{array}{l}\text { Mercoledì } 23 \text { novembre } 2011 \\
\text { dalle } 17.00 \text { alle } 21.00\end{array}$ & $\begin{array}{l}\text { Chiasso } \\
\text { FMH Fiduciaria } \\
\text { Services }\end{array}$ \\
\hline
\end{tabular}

\section{Anmeldung und Auskunft / Inscription et information / Iscrizioni e informazioni} www.fmhservices.ch oder FMH Consulting Services, Cornelia Steinmann, Burghöhe 1, 6208 Oberkirch, Tel. 04192500 77, Fax 0419210586.

\section{Hinweis / Remarque / Osservazioni}

Bei sämtlichen Seminaren, bei denen die Kosten teilweise oder gänzlich von Seminarsponsoren gedeckt werden, werden die Teilnehmeradressen den jeweiligen Sponsoren zur Verfügung gestellt.

Les adresses des participants aux séminaires dont les coûts sont couverts en partie ou totalement par des sponsors sont communiquées aux sponsors concernés. Gli indirizzi dei partecipanti ai seminari, i cui costi sono coperti in parte o completamente da degli sponsor, vengono comunicati agli sponsor interessati.

\section{Annullierungsbedingungen / Conditions} d'annulation / Condizioni d'annullamento

Bei Abmeldungen oder Fernbleiben werden folgende Unkostenbeiträge erhoben:

Un montant est perçu pour une absence ou une annulation. Il est de:

Un importo verrà rimborsato in caso di assenza o annullamento. Esso sarà di:

- 50 CHF pro Person ab 14 Tage vor Seminarbeginn / par personne dans les 15 jours avant le début du séminaire/ per persona entro i 15 giorni prima dell'inizio del seminario;

- 100 CHF pro Person ab 7 Tage vor Seminarbeginn oder Fernbleiben / par personne dans les 7 jours avant le début du séminaire / per persona entro i 7 giorni prima dell'inizio del seminario. 